American Journal of Applied Sciences 5 (9): 1251-1256, 2008

ISSN 1546-9239

(C) 2008 Science Publications

\title{
Dynamic Analysis of Structures Using Neural Networks
}

\author{
${ }^{1}$ N. Ahmadi, ${ }^{2}$ R. Kamyab Moghadas and ${ }^{3}$ A. Lavaei \\ ${ }^{1}$ Department of Civil Engineering, University of Shahid Rajaee, Tehran, Iran \\ ${ }^{2}$ Iranian Academic Center for Education, Culture and Research, Kerman, Iran \\ ${ }^{3}$ Department of Civil Engineering, Islamic Azad University, Borujerd, Iran
}

\begin{abstract}
In the recent years, neural networks are considered as the best candidate for fast approximation with arbitrary accuracy in the time consuming problems. Dynamic analysis of structures against earthquake has the time consuming process. We employed two kinds of neural networks: Generalized Regression neural network (GR) and Back-Propagation Wavenet neural network (BPW), for approximating of dynamic time history response of frame structures. GR is a traditional radial basis function neural network while BPW categorized as a wavelet neural network. In BPW, sigmoid activation functions of hidden layer neurons are substituted with wavelets and weights training are achieved using Scaled Conjugate Gradient (SCG) algorithm. Comparison the results of BPW with those of GR in the dynamic analysis of eight story steel frame indicates that accuracy of the properly trained BPW was better than that of GR and therefore, BPW can be efficiently used for approximate dynamic analysis of structures.
\end{abstract}

Key words: Earthquake, wavelet, wavenet, generalized regression

\section{INTRODUCTION}

As modern digital computers are developed, neural network techniques are considered to use effectively for simplification of complex problems with large number of computations. Dynamic time history analysis of large-scale structures is one of the time consuming problems with complex calculations. In the present study, generalized regression neural networks (GR) and back-propagation wavenet neural networks (BPW) have been employed for approximating of dynamic time history response of an eight stories steel frame structure. Approximating of structural dynamic analysis is very useful in some applications such as optimization. In the neural networks context, two types of activation functions are commonly used: global and local. Global activation functions are active over a large range of input values and provide a global approximation to the empirical data. Local activation functions are active only in the immediate vicinity of the given input value. It is well known that functions can be represented as a weighted sum of orthogonal basis functions. Such expansions can be easily represented as neural networks by having the selected basis functions as activation functions in each hidden neuron and the coefficients of the expansion as the weights on each output neuron. Several classical orthogonal functions, such as sinusoids, sigmoidal functions, etc., but most of them are global approximators and suffer, therefore, from the disadvantages of approximation using global functions. A special class of functions, known as wavelets, possesses good localization properties. Thus, they may be employed as the activation functions of a neural network known as the Wavelet Neural Network or wavenet. Wavenets possess a unique attribute: In addition to forming an orthogonal basis are also capable of explicitly representing the behavior of a function at various resolutions of input variables. The pivotal concept, in the formulation and design of neural networks with wavelets as basis functions, is the multiresolution representation of functions using wavelets. It provides the essential framework for the completely localized and hierarchical training afforded by wavelet neural networks. To create backpropagation wavenet we substitute sigmoidal activation function of hidden layer neurons with a wavelet function. To train GR and BPW a unique training set have been used. Testing of these neural networks indicates that accuracy of BPW is higher than GR and it can be substitute effectively with exact dynamic analysis of structures.

\section{NEURAL NETWORKS}

In the last decade, artificial intelligence techniques have emerged as a powerful tool that could be used to

Corresponding Author: N. Ahmadi, Department of Civil Engineering, University of Shahid Rajaee, Tehran, Iran 
replace time-consuming procedures in many scientific or engineering applications. The interest showed to neural networks ${ }^{[1,2]}$ is mainly due to their ability to process external data and information basing on past experiences. In a neural network the transmission and the processing of the input data are assigned to a network of simple computing units, called neurons. Each neuron returns an output signal when the weighed sum of the inputs exceeds an activation value. The output value is computed by defining a transfer or activation function. The principal advantage of a properly trained neural network is that it requires a trivial computational burden to produce an approximate solution. Such approximations appear to be valuable in situations where the actual response computations are intensive in terms of computing time and a quick estimation is required. For each problem a neural network is trained utilizing information generated from a number of properly selected analyses. The data from these analyses are processed in order to obtain the necessary input and output pairs, which are subsequently used to produce a trained neural network. One of the most important characteristics of neural networks is learning. Learning may be supervised or unsupervised depending on the topology of networks. Therefore, topology, training or learning method and kind of activation function of neurons are its basic characteristics. Neural networks have two operation modes, training mode and normal mode. In the training mode, adjustable parameters of the networks are modified. In the normal mode, the trained networks are applied for simulating of outputs. In various engineering problems, many neural networks ${ }^{[3,4]}$ are widely used.

Generalized regression neural network: Generalized Regression neural network (GR) are powerful and interesting networks due to their rapid training, generality and simplicity. GR that is developed by Specht ${ }^{[5]}$ subsumes the basis function methods. GR is two layers feed forward network. The hidden layer consists of radial basis function neurons with Gaussian activation functions. The response function of neurons of output layer is linear.

First layer of GR has as many neurons as there are input-target vectors in the training set. Weighted input of hidden layer neurons is the distance between the input vector and its weight vector. Output of Each hidden neurons is its input passed through radial basis activation function. The sec layer also has as many neurons as target vectors. The neurons in sec layer act on hidden layer neurons using linear performance function.
This network does not require iterative training therefore training of these networks is very fast. The structure of GR is such designated that transpose of input matrix and transpose of desired output (target) matrix are chosen as first layer and sec layer weight matrixes, respectively. GR algorithm is based on nonlinear regression theory, a well-established statistical technique for function estimation ${ }^{[6]}$. GR network is very good at interpolation.

Back-propagation neural network: Back-Propagation was created by generalizing the Widrow-Hoff learning rule to multiple layer networks and nonlinear differentiable transfer functions. Input vectors and the corresponding target vectors are used to train a network until it can approximate a function, associate input vectors with specific output vectors. Networks with a sigmoid layer and a linear output layer are capable of approximating any function with a finite number of discontinuities.

Standard back-propagation is a gradient descent algorithm, as is the Widrow-Hoff learning rule, in which the network weights are moved along the negative of the gradient of the performance function. The term back-propagation refers to the manner in which the gradient is computed for nonlinear multilayer networks. There are a number of variations on the basic algorithm that are based on other standard optimization techniques, such as conjugate gradient and Newton methods. In this study we have employed Scaled Conjugate Gradient (SCG) algorithm was developed by Moller ${ }^{[7]}$.

The basic back-propagation algorithm adjusts the weights in the steepest descent direction (negative of the gradient). This is the direction in which the performance function is decreasing most rapidly. It turns out that, although the function decreases most rapidly along the negative of the gradient, this does not necessarily produce the fastest convergence. In the conjugate gradient algorithms a search is performed along conjugate directions, which produces generally faster convergence than steepest descent directions. Each of the conjugate gradient algorithms requires a line search at any iteration. This line search is computationally expensive, since it requires that the network response to all training inputs be computed several times for each search. The scaled conjugate gradient algorithm (SCG) was designed to avoid the time-consuming line search.

\section{WAVENETS}

The term wavelet as it implies means a little wave. This little wave must have at least a minimum 
oscillation and a fast decay to zero, in both the positive and negative directions, of its amplitude. This property is analogous to an admissibility condition of a function that is required for the wavelet transform ${ }^{[8]}$.

Sets of wavelets are employed to approximate a signal and the goal is to find a set of daughter wavelets constructed by a dilated and translated original wavelets or mother wavelets that best represent the signal. The daughter wavelets are generated from a single mother wavelet $h(t)$ by dilation and translation:

$$
h_{a, b}(t)=\frac{c}{\sqrt{a}} h\left(\frac{t-b}{a}\right)
$$

where, $\mathrm{a}>0$ is the dilation factor, $\mathrm{b}$ is the translation factor and $\mathrm{c}$ is correction factor ${ }^{[9]}$.

Wavelet neural networks employing wavelets as the activation functions recently have been researched as an alternative approach to the neural networks with sigmoidal activation functions.

The combination of wavelet theory and neural networks has lead to the development of wavelet networks. Wavelet networks are feed-forward neural networks using wavelets as activation function. In wavelet networks, both the position and the dilation of the wavelets are optimized besides the weights.

Wavenet is another term to describe wavelet networks. Originally, wavenets did refer to neural networks using wavelets. In wavenets, the position and dilation of the wavelets are fixed and the weights are optimized $^{[10]}$.

Back-propagation wavenet: Back-Propagation (BP) neural network is now the most popular mapping neural network. But BP neural network has few problems such as trapping into local minima and slow convergence. Wavelets are a powerful tool for signal analysis. They can approximately realize the time-frequency analysis using a mother wavelet. The mother wavelet has a square window in the time-frequency space. The size of the window can be freely variable by two parameters. Thus, wavelets can identify the localization of unknown signals at any level. Activation function of hidden layer neurons in back-propagation network is a sigmoidal function shown in Fig. 1a. This type of activation function provides a global approximation on the search space.

In this study we have substituted hidden layer sigmoidal activation function of back-propagation neural network with POLYWOG1 wavelet ${ }^{[9]}$ :

$$
\mathrm{h}_{\text {POLYWOG1 }}(\mathrm{t})=\sqrt{\mathrm{e}} \cdot(\mathrm{t}) \cdot \mathrm{e}^{\left(-(\mathrm{t})^{2}\right) / 2}
$$

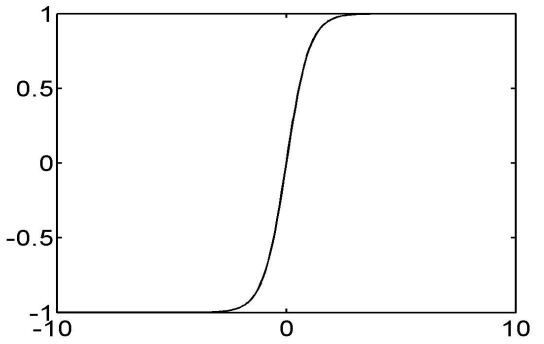

(a)

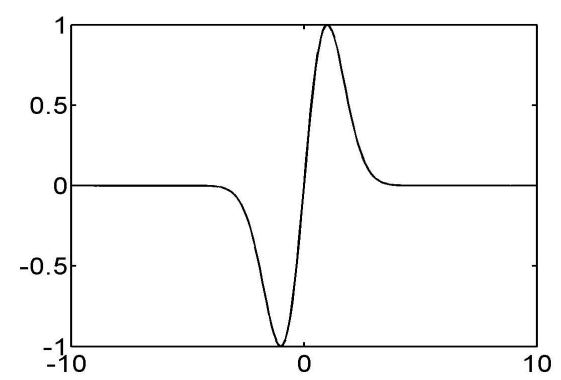

(b)

Fig. 1: (a): Sigmoidal function, (b): POLYWOG mother wavelet

Diagram of POLYWOG1 with $a=1$ and $b=0$ is shown in Fig. $1 \mathrm{~b}$.

This type of activation function provides a local approximation to the experimental data. In backpropagation wavenets (BPW), the position and dilation of the wavelets as activation function of hidden layer neurons are fixed and the weights of network are optimized using scaled conjugate gradient (SCG) algorithm. In this study we suppose $\mathrm{a}=2$ and $\mathrm{b}=0$.

$$
\mathrm{h}_{\text {POLYWOG1 }}(\mathrm{t})=\sqrt{\mathrm{e}} \cdot\left(\frac{\mathrm{t}}{2}\right) \cdot \mathrm{e}^{\left(-\left(\frac{\mathrm{t}}{2}\right)^{2}\right) / 2}
$$

Therefore, BPW is a modified back-propagation neural network with local approximation property and POLYWOG1 hidden layer neurons activation function. And adjusting the weights of network are done Using Scaled Conjugate Gradient (SCG) algorithm. Structure of BPW is shown in Fig. 2.

\section{NUMERICAL RESULTS}

Methodology: The eight story steel frame structure that shown in Fig. 3, has been considered as this study model. In this model, rigid diaphragms are assigned to the roofs. Cross sections of columns and beams are 


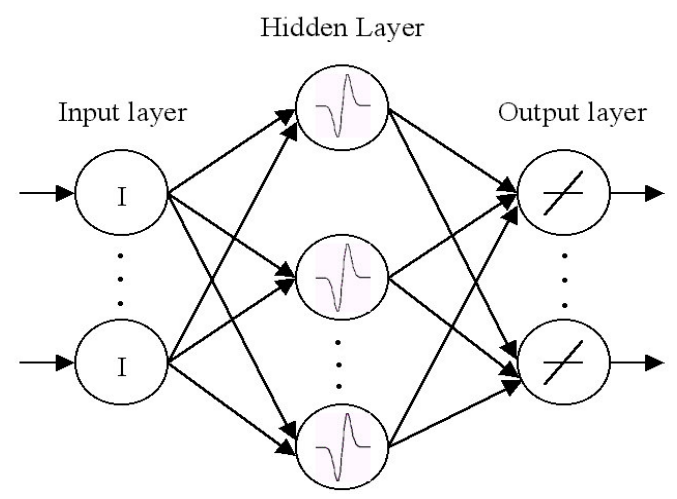

Fig. 2: Structure of BPW

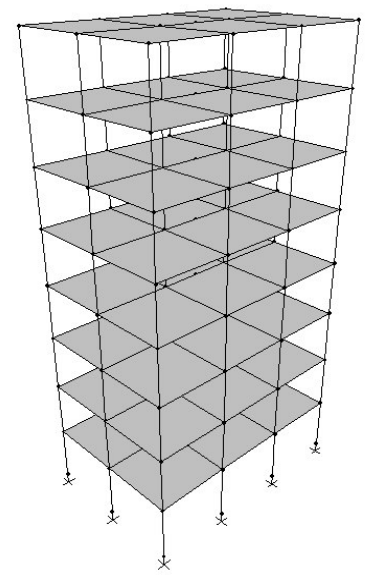

Fig. 3: Eight story steel frame structure

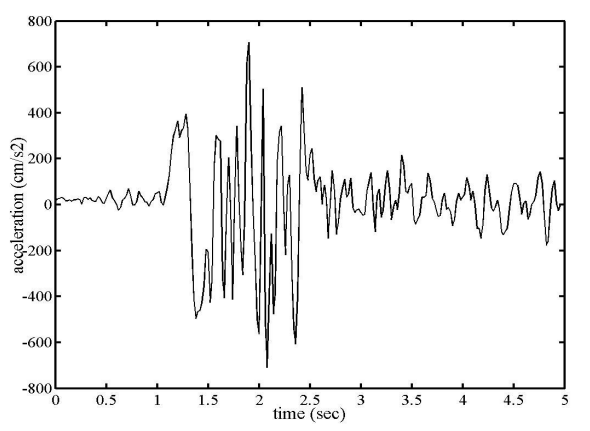

Fig. 4: Naghan earthquake 1977 (Iran)

selected from the wide flange sections available in european profile list. The model was subjected to seismic load. This seismic loading that consists of Naghan earthquake 1977 (IRAN), shown in Fig. 4, is effectively used in $\mathrm{x}$ direction. Spans in $\mathrm{x}$ and $\mathrm{y}$
Table 1: Cross sections

\begin{tabular}{llc}
\hline Code & Columns & Beams \\
\hline 1 & HE 500-M & \\
2 & HE 600-M & 2IPE 500 \\
3 & HE 700-M & \\
4 & HE 800-M & \\
\hline
\end{tabular}

Table 2: Grouping of elements

\begin{tabular}{ll}
\hline Group & Elements \\
\hline 1 & Story 1,2 columns \\
2 & Story 3,4 columns \\
3 & Story 5,6 columns \\
4 & Story 7,8 columns \\
\hline
\end{tabular}

Table 3: Test vectors

\begin{tabular}{lllll}
\hline & Cross section & & & \\
No. & Group 1 & Group 2 & Group 3 & Group 4 \\
\hline 1 & HE 600-M & HE 600-M & HE 600-M & HE 500-M \\
2 & HE 700-M & HE 600-M & HE 500-M & HE 500-M \\
3 & HE 700-M & HE 700-M & HE 600-M & HE 500-M \\
4 & HE 700-M & HE 600-M & HE 600-M & HE 600-M \\
5 & HE 800-M & HE 600-M & HE 600-M & HE 500-M \\
6 & HE 800-M & HE 700-M & HE 600-M & HE 600-M \\
7 & HE 800-M & HE 700-M & HE 700-M & HE 600-M \\
8 & HE 800-M & HE 800-M & HE 700-M & HE 700-M \\
9 & HE 800-M & HE 800-M & HE 800-M & HE 600-M \\
\hline
\end{tabular}

directions are $4 \mathrm{~m}$. Height of each story is $3 \mathrm{~m}$. the sum of dead load and live load is $500 \mathrm{~kg} \mathrm{~m}^{-2}$ and masses are calculated from loads. Analysis is performed using SAP2000 developed by Wilson ${ }^{[1]]}$.

In order to simplify the analysis, 4 types of cross sections are considered for the columns and a unique one is considered for all beams that are shown in Table 1. Due to practical demands the columns are divided into 4 groups, shown in Table 2, having the same cross-sections. In the present study, we train GR and BPW neural networks for approximating the last story time history response in $\mathrm{x}$ direction, using $\mathrm{MATLAB}^{[12]}$. To approximae the time history responses of the other stories, the same procedure can be used. A total number of 39 structures are generated and analyzed from which 30 structures are used for training and 9 pairs are employed for testing the networks which are shown in Table 3.

Dynamic analysis using neural networks: Trained GR and BPW neural networks are employed for approximating of the last story time history response. The results of testing the networks are shown in Fig. 5-10 for three test samples. Similar results exist for the other test samples. As shown in these Fig. accuracy of BPW is much better than that of GR, furthermore, the accuracy of GR results in the case of some test vectors, such as 5 to 9 th test vectors, are very poor. 


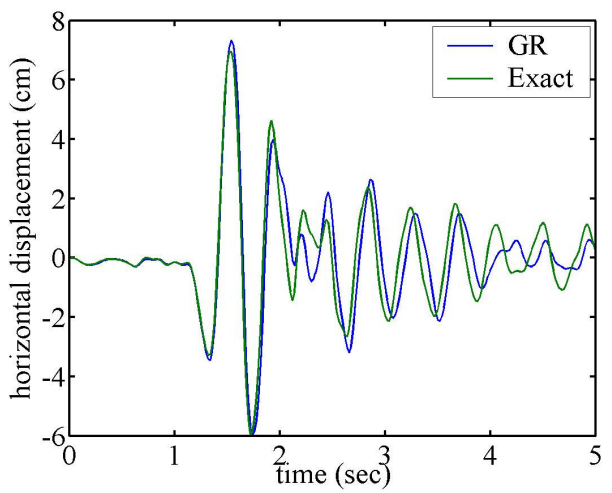

Fig. 5: 7th approximate response by GR

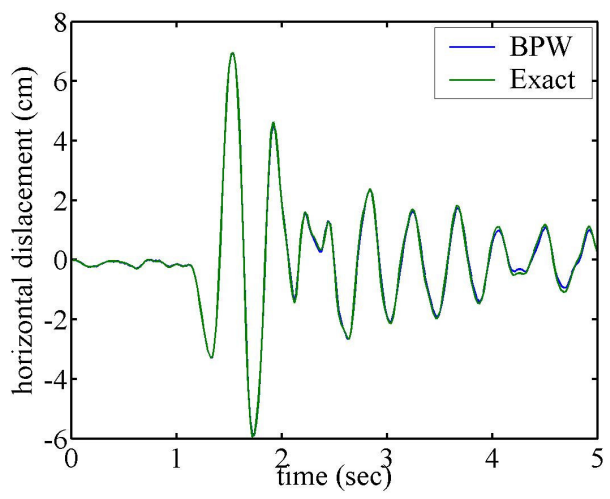

Fig. 6: 7th approximate response by BPW

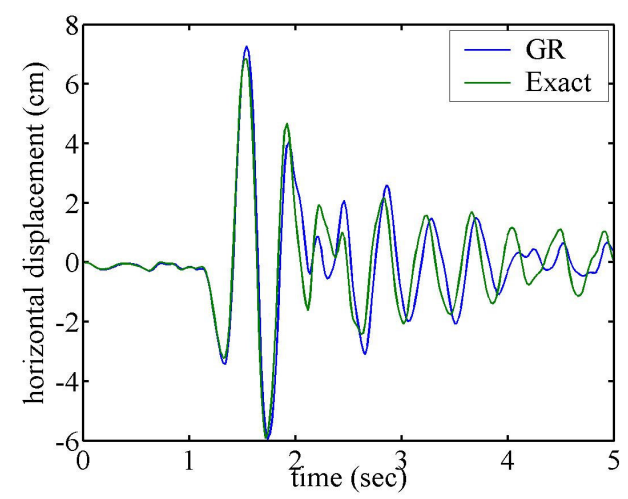

Fig. 7: 8th approximate response by GR

Therefore, GR cannot be effectively employed for approximating the time history response of frame structures.

To present the BPW network results, the approximate responses of last story of the frame structure are divided to five intervals. These intervals are considered as the first to fifth sec of the response

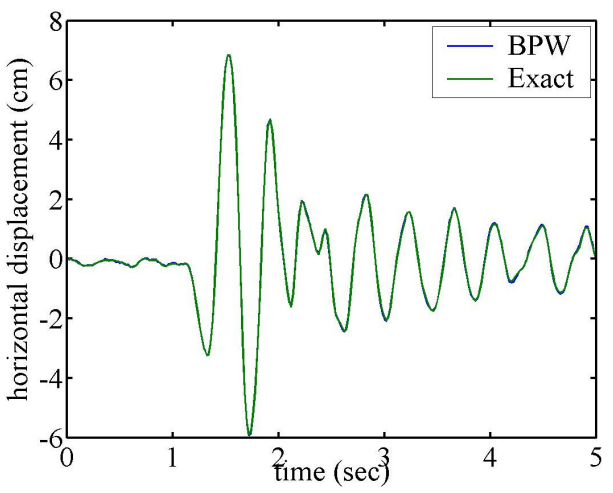

Fig. 8: 8th approximate response by BPW

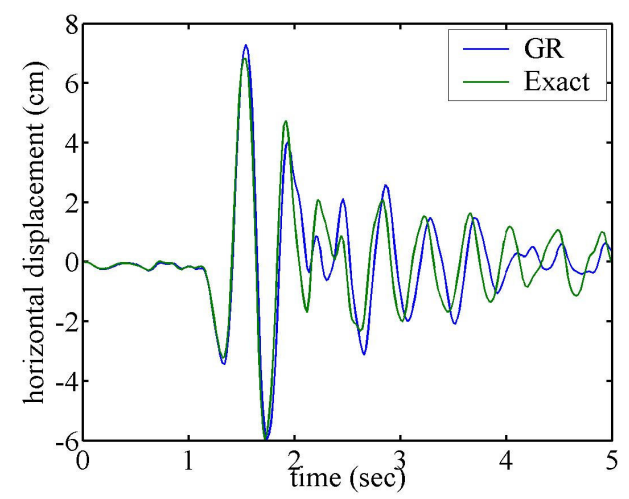

Fig. 9: 9th approximate response by GR

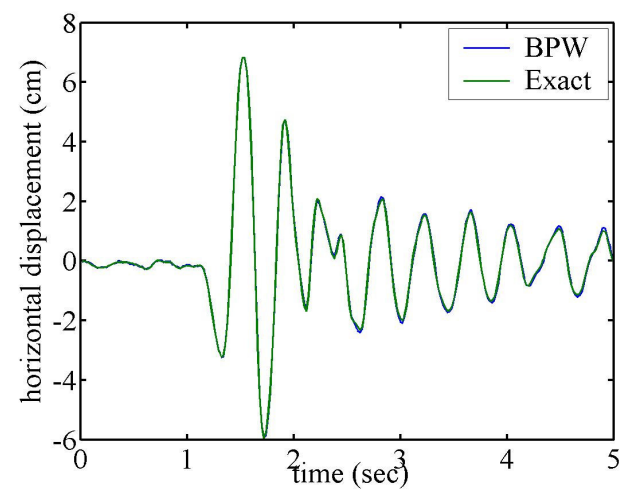

Fig. 10: 9th approximate response by BPW

curves. Average error in each interval is shown in Table 4.

As shown in Table 4, the average of error in the first to fourth sec of all approximate time history response is less than 5\%. While the average error in the fifth sec experiments its maximum values. 
Table 4: Average error of BPW approximate results

\begin{tabular}{llllll}
\hline & Average error (\%) & & & \\
No. & Second 1 & Second 2 & Second 3 & Second 4 & Second 5 \\
\hline 1 & 4.799 & 0.082 & 0.585 & 4.087 & 10.34 \\
2 & 1.703 & 0.151 & 0.957 & 2.449 & 9.546 \\
3 & 2.476 & 0.524 & 3.254 & 3.196 & 8.904 \\
4 & 3.180 & 0.545 & 3.833 & 3.798 & 8.650 \\
5 & 2.439 & 0.237 & 4.465 & 3.586 & 8.613 \\
6 & 1.586 & 0.643 & 1.284 & 4.492 & 9.429 \\
7 & 1.247 & 0.605 & 1.028 & 4.241 & 6.146 \\
8 & 0.662 & 0.275 & 0.103 & 2.702 & 5.251 \\
9 & 0.441 & 0.326 & 0.473 & 3.514 & 5.511 \\
\hline
\end{tabular}

These results indicate that the properly trained BPW network has acceptable accuracy and can be effectively used for approximating the time history response of frame structures.

\section{CONCLUSION}

Computational burden of dynamic analysis of frame structures for earthquake loads, is usually very high. In order to reduce the computational work, one of the best choices is neural networks. We have employed two various kinds of neural networks: Generalized Regression (GR) networks as a traditional neural network and Back-Propagation Wavenet (BPW) network as a wavelet neural network for approximating the dynamic time history response of frame structures. Approximation strategy in GR network is global and in BPW network is local. Comparison of GR and BPW networks results in the approximation of dynamic time history response of frame structures against the earthquake indicates that BPW network using local approximation strategy is a powerful network with high accuracy. While GR network that employ global approximation strategy cannot present proper accuracy. Therefore, BPW network using wavelets as activation function of back-propagation neurons is a powerful tool for approximating of dynamic analysis of structures.

\section{REFERENCES}

1. Rafiq, M.Y., G. Bugmann and D.J. Easterbrook, 2001. Neural Network Design for Engineering Applications. Comput. Struct., 79 (17): 1541-1552.

2. Waszczyszyn, Z. and L. Ziemianski, 2001. Neural Networks in Mechanics of Structures and Materials-New Results and Prospects of Applications. Comput. Struct., 79 (22): 2261-2276.

3. Zhang, A. and L. Zhang, 2004. RBF Neural Networks for the Prediction of Building Interference Effects. Comput. Struct., 82: 2333-2339.

4. Jiang, N., Z. Zhao and L. Ren, 2003. Design of Structural Modular Neural Networks with Genetic Algorithm. Adv. Eng. Software, 34: 17-24.

5. Specht, D.F., 1991. A General Regression Neural Networks. Trans. Neural Networks, 2: 568-576.

6. Wasserman, P.D., 1993. Advanced methods in neural computing. Prentice Hall Company, Van Nostrand Reinhold, New York.

7. Moller, M.F., 1993. A Scaled Conjugate Gradient Algorithm for Fast Supervised Learning. Neural Networks, 6: 525-533.

8. Young, R.K., 1993. Wavelet theory and its applications. Kluwer Acadamic Publishers, Boston, MA.

9. Lekutai, G., 1997. Adaptive self-tuning neuro wavelet network controllers. ph.D thesis, Virginia Polytechnic Institute and State University, The Electrical Engineering Department.

10. Thuillard, M., 2000. A review of wavelet networks, wavenets, fuzzy wavenets and their applications. ESIT, Aachen, Germany.

11. Wilson, E., 2000. Structural Analysis Program SAP2000. Berkeley, California.

12. The Language of Technical Computing, MATLAB, Math Works Inc. 2001. 